\title{
STRONG CONSISTENCY OF LS ESTIMATORS IN SIMPLE LINEAR EV REGRESSION MODELS WITH WOD ERRORS
}

\author{
Yi Yanchun, Pingyan Chen And Soo HaK Sung*
}

Abstract. For a simple linear errors-in-variables regression model with widely orthant dependent errors, we provide sufficient conditions for the convergence rate in the strong consistency of the least squares estimators. We also provide necessary conditions. Our result improves and extends some results of Liu et al. (J. Math. Ineq., 14 (2020), 771-779).

Mathematics subject classification (2020): 62F12, 60F15.

Keywords and phrases: Strong consistency, simple linear errors-in-variables regression model, widely orthant dependent random variable.

\section{REFERENCES}

[1] P. CHEN, AND S. H. Sung, A Spitzer-type law of large numbers for widely orthant dependent random variables, Statist. Probab. Lett., 154 (2019), 108544.

[2] P. CHen, L. WEN AND S. H. Sung, Strong and weak consistency of least squares estimators in simple linear EV regression models, J. Statist. Plann. Infer., 205 (2020), 64-73.

[3] P. CHEN, T. ZHANG AND S. H. Sung, Strong laws for randomly weighted sums of random variables and applications in the bootstrap and random design regression, Stat. Sinica, 29 (2019), 1739-1749.

[4] A. Deaton, Panel data from a time series of cross-sections, J. Econometrics, 30 (1985), 109-126.

[5] W. Fuller, Measurement Error Models, Wiley, New York, 1987.

[6] D. Hu, P. Chen And S. H. Sung, Strong laws for weighted sums of $\psi$-mixing random variables and applications in errors-in-variables regression models, TEST, 26 (2017), 600-617.

[7] J. LAng, T. HE, Z. YU, Y. WU AND X. WANG, Complete convergence for randomly weighted sums of random variables and its application in linear-time-invariant systems, Comm. Statist. Simul. Comput., (2021), doi:10.1080/03610918.2020.1870695.

[8] J. LITA DA SILVA, Almost sure convergence for weighted sums of pairwise PQD random variables, arXiv:1812.09947 [math.ST] (2018).

[9] J. LiTA DA SILVA, Almost sure convergence for weighted sums of $\phi$-mixing random variables with applications, Comm. Statist. Theory Methods, 49 (2020), 3882-3894.

[10] J. LiTA DA SiLVA, Strong laws of large numbers for arrays of row-wise extended negatively dependent random variables with applications, J. Nonparametr. Stat., 32 (2020), 20-41.

[11] J. LIU AND X. CHEN, Consistency of LS estimator in simple linear EV regression models, Acta Math. Sci., Ser. B, Engl. Ed., 25 (2005), 50-58.

[12] X. LiU, X. Li, W. JiAng AND F. FU, Strong consistency of LS estimator in simple linear EV regression models, J. Math. Ineq., 14 (2020), 771-779.

[13] Y. Miao, K. WANG AND F. Zhao, Some limit behaviors for the LS estimator in simple linear EV regression models, Stat. Probab. Lett., 81 (2011), 92-102.

[14] X. WAng, A. Shen, Z. Chen AND S. Hu, Complete convergence for weighted sums of NSD random variables and its application in the EV regression model, TEST, 24 (2015), 166-184.

[15] K. WANG, Y. WANG AND Q. GAO, Uniform asymptotics for the finite-time ruin probability of a dependent risk model with a constant interest rate, Methodol. Comput. Appl. Probab., 15 (2013), 109-124.

[16] X. WANG, Y. WU AND S. HU, Strong and weak consistency of LS estimators in the EV regression model with negatively superadditive-dependent errors, AStA Adv. Stat. Anal., 102 (2018), 41-65. 
[17] Y. WU, X. WANG, S. HU AND L. YANG, Weighted version of strong law of large numbers for a class of random variables and its applications, TEST, 27 (2018), 379-406.

[18] Y. Yi, P. CHEN AND S. H. SUnG, Strong laws for weighted sums of random variables satisfying generalized Rosenthal type inequalities, J. Ineq. Appl., 2020 (2020), 43.

[19] M. Zhang, P. CHEN AND S. H. Sung, Convergence rates in the weak law of large numbers for weighted sums of i.i.d. random variables and applications in errors-in-variables models, Stoch. Dynam., 19 (2019) 1950041. 